\title{
Die Kluft zwischen Handlungsmotiven und Handlungsergebnissen - Der ordonomische Blick auf die Flüchtlingsdebatte
}

\section{Kommentar zum Hauptbeitrag von Ingo Pies}

\section{Zielsetzung des Beitrags}

[1] Im Zentrum der Kritik an der Ökonomik steht zumeist die Kunstfigur des Homo Oeconomicus, der als Nutzenmaximierer vorgeblich über ein zu beschränktes Motivationsspektrum verfügt. Dabei gerät leicht eine Grunderkenntnis der Ökonomik aus dem Blick, die den Dreh- und Angelpunkt des Beitrags von Ingo Pies darstellt: Unabhängig von der Art der individuellen Motivationslagen kommt es bei sozialen Interaktionen vielfach zu einem »Hiatus zwischen den Handlungsmotiven einzelner Akteure (...) und ihren Handlungsergebnissen « ([5]). Diese Kluft manifestiert sich in gegensätzlicher Weise: Einerseits können moralisch eher negativ bewertete Handlungsgründe (`Egoismus`) auf gesellschaftlicher Ebene durchaus positive Auswirkungen (>Wohlfahrtsmaximierung $<$ ) zeitigen, andererseits können höchst ehrenwerte Motive (’Hilfsbereitschaft $<$ ) negative Folgen haben (,Wohlfahrtsverluste durch Aushöhlung von Leistungsbereitschaft‘). Das klassische Beispiel für eine Kluft der ersten Art liefert uns das bekannte Metzger-Brauer-Bäcker-Gleichnis von Adam Smith, in dem sich bereits die Grundidee des in einer idealen Marktökonomie gültigen 'Ersten Hauptsatzes der Wohlfahrtstheorie andeutet. Im zweiten Fall wird man schnell an Kurt Tucholskys bekannte Redewendung »Das Gegenteil von >gut ist 'gut gemeint« « erinnert.

[2] Die empirische Relevanz des zweiten Hiatus-Typs wird von der Ökonomik weniger systematisch erörtert als die des ersten, weil 'good intentions with bad outcomes eher auf politischer Ebene als in Marktprozessen anzutreffen sind. Die Arbeit von Ingo Pies lässt sich vor diesem Hintergrund als Versuch interpretieren, diese Lücke zu schließen und dem zweiten Hiatus-Typ die gebührende Beachtung zu schenken. Sein ordonomischer Ansatz greift dabei über den gewohnten Denkrahmen der Ökonomik weit hinaus und nimmt neben Markt- und Politikversagen auch das "Diskursversagen « in der öffentlichen Meinungsbildung mit in den Blick (vgl. [8]). Dabei verfolgt Ingo Pies insbesondere die Absicht, durch Formulierung von »Überbietungsargumenten « zur Überwindung von Denk- und Diskursblockaden, zur Stimulierung von Lernprozessen und damit zur Verbesserung

* Prof. Dr. Wolfgang Buchholz, Institut für Volkswirtschaftslehre und Ökonometrie, Universität Regensburg, Universitätsstraße 31, D-93053 Regensburg, Tel. +49(0)941-9432711, E-Mail: wolfgang.buchholz@ur.de, Forschungsschwerpunkte: Umweltökonomie, Sozialpolitik, Wirtschaftsethik. Für hilfreiche Kommentare möchte ich mich bei Peter Heindl und Dirk Rübbelke bedanken. 
der zivilgesellschaftlichen Kommunikation beizutragen (vgl. [7]). Insbesondere sucht er dabei nach Mitteln und Wegen, um gegensätzlichen normativen Positionen gleichzeitig Rechnung zu tragen und somit - bildlich gesprochen - »überbieten « zu können. Wie eine solche >Aufhebung argumentativer Gegensätze konkret aussehen kann, demonstriert Ingo Pies dann am Beispiel der aktuellen Flüchtlingsdebatte in Deutschland. Bevor ich in Abschnitt 3 auf diese empirische Thematik näher eingehe, möchte ich in Abschnitt 2 die auch ideengeschichtlich höchst interessanten theoretischen Grundlagen der Argumentation von Ingo Pies reflektieren, wobei ich auf Verbindungen zu einigen neueren Ansätzen in der Ökonomik eingehe.

\section{Theoretische Grundlagen}

[3] Die theoretischen Überlegungen (vgl. [22]ff.) starten mit einer Interpretation des bekannten Gefangenen-Dilemmas. ${ }^{1}$ Dabei bedient sich Ingo Pies höchst erhellender Kategorisierungen, die schon vor mehr als 350 Jahren von Thomas Hobbes (vgl. 1996 [1651]) entwickelt wurden. So trifft Hobbes mit dem Verweis auf das Naturrecht eine grundsätzliche Unterscheidung zwischen Verpflichtungen in foro interno<, d.h. solchen, die im Hinblick auf die Mehrung des allgemeinen Wohls und somit aus Gründen der kollektiven Rationalität angemessen sind, und solchen in foro externo<, die sich in einer gegebenen Situation individuell als rational und unter Umständen sogar als überlebenswichtig erweisen (vgl. [32]). Genauso, wie es ein natürliches Gebot der Vernunft ist, zur Vermeidung von Leid (auf der gedanklichen Ebene der Formulierung von Handlungszielen) stets allseitigen Frieden anzustreben, hat man zum Schutz der eigenen Unversehrtheit (auf der Ebene konkreten Handelns) ein natürliches Recht auf Verteidigung, wenn man von anderen angegriffen wird. Eine absolute und unbedingte Pflicht zu friedlichem Verhalten (oder ganz analog zur Kooperation bei der Bereitstellung eines öffentlichen Gutes) besteht somit nicht, sondern nur eine bedingte, bei der die Konditionalität vom Verhalten der anderen abhängt: Wenn die anderen nicht friedlich bzw. unkooperativ sind, ist man selber auch nicht zur Friedfertigkeit bzw. zu kooperativen Anstrengungen verpflichtet - for that were to expose himself to prey (which no man is bound to) (Hobbes 1996 [1651]: 87, und [29]). Um das Wohl aller zu mehren und Ausbeutung zu verhindern, postuliert Hobbes somit reziprokes Verhalten gemäß dem Motto 'Wie du mir, so ich dir`, auf dem auch der freiwillige Markttausch von Gütern beruht. Wenn dabei alle Beteiligten die kooperative Strategie wählen, finden forum internum und forum externum zusammen.

[4] Eine funktionierende Kooperation zum Wohle aller ergibt sich in einer Welt unabhängig agierender Agenten nicht von selber. Vielmehr werden zur Regelung der Kooperationsbeziehungen Verträge erforderlich, denen oftmals langwierige

1 Zur näheren Beschreibung und Diskussion des Gefangenen-Dilemmas vgl. etwa Sandler/ Arce M. (2005) und Buchholz/Eichenseer (2017). 
Verhandlungen vorausgehen. Auch nach Abschluss eines Vertrages ist dessen Einhaltung keineswegs gesichert, weil in einer Gefangenen-Dilemma-Konstellation die Vertragspartner einen Anreiz zum Bruch der Vereinbarung haben. Nach Hobbes lässt sich vertragstreues Verhalten nur durch die Furcht vor Sanktionen erreichen, die sowohl psychologischer (= Furcht vor der Strafe Gottes) als auch realer Natur (= Furcht vor von Menschen ausgeübter Zwangsgewalt) sein können (vgl. Hobbes, 1996 [1651]: 87).

[5] Die Beziehung zwischen forum internum und forum externum wird im Rahmen des ordonomischen Ansatzes anhand einer Transformation des ursprünglichen Gefangenen-Dilemma-Spiels (vgl. [34]-[36]) in ein Metaspiel (auf der Verhandlungsebene mit den im Wesentlichen gleichen Kooperationshemmnissen wie im Basisspiel (vgl. [35])) und ein Meta-Meta-Spiel (auf der Wahrnehmungs- und Meinungsbildungsebene (vgl. [36] und Fußnote 10)) näher erklärt und präzisiert. Durch Orientierung am Reziprozitätsprinzip im Sinne einer »bedingten Kooperations-Strategie « (Fußnote 10) verwandelt sich dabei das Gefangenen-DilemmaSpiel in ein Koordinationsspiel, bei dem Freifahrerverhalten ausgeschlossen ist. Eine effektive Kommunikation auf der Meta-Meta-Ebene führt zum Erfolg der Verhandlungen auf der Meta-Ebene. Wenn dabei Sanktionsmechanismen vereinbart werden, kann die kooperative Lösung auf der Ebene des Basis-Spiels implementiert werden.

[6] Mit seiner Bezugnahme auf Spieltransformationen an zentraler Stelle seiner theoretischen Ausführungen trifft sich Ingo Pies mit spieltheoretischen Ansätzen, die in den letzten Jahren entwickelt worden sind. Dabei werden die materiellen Pay-offs des Basis-Spiels durch psychologische Präferenzkomponenten ergänzt, in denen nicht nur der Wunsch nach Gleichverteilung der Ergebnisse, sondern beginnend mit Rabin (1993) - auch das Verlangen nach Fairness und Reziprozität zum Ausdruck kommen kann (vgl. Pittel/Rübbelke 2013; Buchholz et al. 2014; Ufert 2016). Solche erweiterten Präferenzen ${ }^{2}$ und die von ihnen bewirkten Spieltransformationen liefern nicht nur eine passende Ergänzung zum ordonomischen Drei-Ebenen-Schema, sondern erfassen auch die Wirkung der psychologischen Sanktionsmechanismen, in denen Hobbes (s.o.) ein Mittel zur Stabilisierung vertraglicher Kooperationsbeziehungen sieht. In diesem Zusammenhang würde man heute allerdings eher von sschlechtem Gewissen als von der `Furcht vor der Strafe Gottes< sprechen.

[7] Auf einen mit dem ordonomischen Ansatz verwandten Grundgedanken treffen wir auch bei den neueren, an Kantischen Konzepten orientierten spieltheoretischen Ansätzen (vgl. Roemer 2015; van Long 2017), in deren Rahmen die Spieler ihre optimalen Strategien anders als bei den üblicherweise verwendeten NashReaktionen nicht aus einer rein ich-bezogenen ('autarken`) Perspektive bestimmen. Stattdessen überlegen sie sich in Kantischer Manier, was für sie das beste Ergebnis wäre, falls die anderen Spieler genauso wie sie selber handeln. Beispiels-

2 Eine verwandte Idee, gemäß der Individuen ihre Handlungen (etwa bei freiwillig vorgenommenen Recycling-Aktivitäten) an moralischen Vorstellungen ausrichten und auf diese Weise sozial verantwortlich handeln, findet sich bei Brekke et al. (2003). 
weise würde ein Spieler seinen Beitrag zu einem öffentlichen Gut nur dann um ein Prozent vermindern wollen, falls sich dies für ihn auch dann lohnt, wenn gleichzeitig auch alle anderen Individuen eine solche einprozentige Reduktion vornehmen.

[8] Die Orientierung am Reziprozitätsprinzip kommt bei einer solchen `Kantischen Optimierung ^ nicht durch die Modifikation von Präferenzen, sondern durch die Veränderung des individuellen Entscheidungskalküls zustande, die einer spezifischen moralischen Norm, eben der universalistischen Ethik Kants, entspringt. Die Kantische Optimierung beruht auf einem Gedankenexperiment und bewegt sich deshalb ausschließlich in der Sphäre des 'forum internum ‘. Der »Hiatus zwischen Denken und Handeln, der erst durch die Schaffung eines institutionellen Arrangements (...) überwunden werden muss, «([40]) bleibt deshalb bestehen.

[9] Dass sich in der Realität viele Individuen auch ohne Absicherungs- und Sanktionsmechanismen bis zu einem gewissen Grade konditional kooperativ verhalten und in dieser Weise einem intrinsischen Reziprozitätsprinzip folgen, ändert nur wenig an der fundamentalen Einsicht, dass kooperative Beziehungen in der Regel einer institutionellen Absicherung bedürfen. Bei der in einer Vielzahl ökonomischer Experimente beobachteten konditionalen Kooperationsbereitschaft ${ }^{3}$ spielt überdies die Kommunikation in Verhandlungssituationen keine allzu große Rolle. In seiner Auseinandersetzung mit der Flüchtlingsproblematik stellt Ingo Pies gerade diesen Aspekt in den Vordergrund.

\section{Die Flüchtlingsdebatte}

[10] Um in der Flüchtlingsdebatte die »den Diskurs blockierende Ziel-MittelKonfusion " ([43]) und die ihr zugrundeliegende »dysfunktionale(n) (Ver-)Wendung der Moralkommunikation « ([45]) zu überwinden, formuliert Ingo Pies in Anwendung der ordonomischen Überbietungsstrategie ein Konzept, das sowohl die normative Position der Flüchtlingsbefürworter als auch die der Flüchtlingsgegner zu berücksichtigen versucht. In dieser »diskursiven Konsenszone(n) « ([52]) finden sich Vorschläge zur Einrichtung von durch die EU finanzierten und kontrollierten Auffanglagern in Afrika, zur Schaffung von Einreisemöglichkeiten in die EU (als Flüchtling oder als Arbeitsmigrant) durch Antragstellung bereits im Herkunftsland (vgl. [55]) sowie zur Bekämpfung der Fluchtursachen durch Verbesserung der Lebensumstände in den Fluchtregionen (vgl. [62]). Durch solche Maßnahmen soll dafür gesorgt werden, dass die humanitäre Hilfe zielgerichteter, effektiver und kosteneffizienter oder im besten Falle sogar entbehrlicher wird. ${ }^{4}$ Über jeden dieser Vorschläge ließe sich im Detail lange diskutieren, was teilweise

3 Als Klassiker der experimentellen Studien zur konditionalen Kooperation gilt Fischbacher et al. (2001). Zu reziprokem Verhalten in verschiedenen empirischen Kontexten vgl. auch Dohmen et al. (2009).

4 Vgl. zu Vorschlägen diese Art auch Betts/Collier (2017).

zfwu, 18/2 (2017) 
schon geschieht. ${ }^{5}$ Hier möchte ich mich jedoch auf die Erörterung einer grundsätzlichen Problematik beschränken, die sich gerade im Zusammenhang mit der Flüchtlingskrise und ihrer medialen Wahrnehmung deutlich gezeigt hat. Ingo Pies spricht diesen Punkt gegen Ende seines Beitrages kurz an, indem er Mindeststandards für politische Diskurse fordert, deren Einhaltung durch gezielte Maßnahmen (wie die Vermittlung von Medienkompetenz im Schulunterricht) abgesichert werden soll. Zumindest im Jahr 2015, in dem so viel von Obergrenzen (für Flüchtlingszahlen) die Rede war, wurde in der Flüchtlingsdebatte eine Untergrenze für das intellektuelle Diskursniveau klar unterschritten, was sowohl allgemeine als auch situationsspezifische Gründe hatte.

[11] Zum Verständnis der allgemeinen Gründe für das Diskursversagen in der Flüchtlingsdebatte kann man aus der Perspektive der »Ökonomischen Theorie der Politik « auf den »Issue-Attention-Zyklus « Bezug nehmen (vgl. Downs 1972), der ursprünglich zur Erklärung eines bestimmten zeitlichen Ablaufmusters in der Umweltpolitik entwickelt wurde. Damit ist gemeint, dass bei Auftreten eines politischen Problems mit hohem Betroffenheitspotential zunächst mit einer Phase semotionaler Euphorie zu rechnen ist, in der auf die erst später anfallenden Kosten monetärer und nicht-monetärer Art der jeweiligen Politikmaßnahme nur wenig geachtet wird. Erst wenn nach einiger Zeit diese Kosten konkret spürbar werden, tritt Ernüchterung ein, und die ursprünglich allseits begrüßte Maßnahme wird zunehmend kritisch gesehen. In der Flüchtlingskrise folgte auf die >Willkommenskultur des Sommers und Herbsts 2015 gemäß diesem Muster die Desillusionierung nach der Kölner Silvesternacht.

[12] Man kann den Erklärungsansatz von Downs mit psychologischen Argumenten ergänzen. So ist ein emotionaler Überschwang besonders dann wahrscheinlich, wenn an Mitleid und moralische Verantwortung (= Verpflichtung zur Nothilfe) appelliert wird und man sich in die Leidenssituation der hilfsbedürftigen Menschen (= Familien mit kleinen Kindern) gut einfühlen kann. ${ }^{6} \mathrm{Zu}$ Recht kann aber bezweifelt werden, dass Empathie ein guter Ratgeber für rationale moralische Urteile und Entscheidungen ist, wie etwa Bloom (2016: 31) bemerkt: «Empathy is like a spotlight (...). Its spotlight nature renders it innumerate and myopic (...), and it is insensitive to statistical data and estimated costs and benefits."

[13] Das Spannungsverhältnis zwischen Gefühls- und Verstandesethik ist gerade in der Flüchtlingsfrage hochkomplex und intellektuell herausfordernd ${ }^{7}$. Als auf die eigene Karriere bedachte* $\mathrm{r}$ Journalist*in ist man auf der sichereren Seite, wenn man sich von moralischen Konflikten fernhält und die Position der Gesin-

5 Zur Forderung nach einer Visa-Erteilung aus humanitären Gründen hat sogar der Europäische Gerichtshof (EuGH) kürzlich Stellung genommen und die Klage einer syrischen Familie abschlägig beschieden. Vgl. zur moralisch motivierten Kritik an diesem Urteil z.B. Janisch (2017).

6 Wie in der Flüchtlingskrise die deutschen Leitmedien eine solche Gefühlslage förderten, beschreibt Alexander (2017: insbes. 69f.).

7 Eine Übersicht über die kontroversen Positionen liefert der Sammelband von Grundmann/Stephan (2016), in dem insbesondere die für eine umfassende ethische Diskussion wichtige quantitative Dimension der Flüchtlingsthematik ausführlich behandelt wird. 
nungsethiker mit ihren hehren Idealen und einfachen Antworten einnimmt. Anspruchsvolle ethische Kontroversen, wie sie im Hinblick auf die Flüchtlingsthematik zu führen wären, eignen sich kaum für Talk-Shows. Die in der medialen Vermittlung typischen Vereinfachungen befördern jedoch fast zwangsläufig »das Denken in Kategorien von Gut und Böse sowie das Denken in Freund-Feind-Schemata « ([72]). Auf diese Weise lässt sich das von Ingo Pies beklagte Versagen des öffentlichen Diskurses in der Flüchtlingsdebatte auch durch die Anreizmechanismen erklären, die den Medienbetrieb beherrschen.

[14] Im Hinblick auf die situationsspezifischen Faktoren sind insbesondere zu nennen: Der hohe Stellenwert verfestigter gesinnungsethischer Vorstellungen in der öffentlichen Meinung; die historisch bedingte Abneigung gegenüber rechten politischen Positionen und ‘Dunkeldeutschland ‘ das gewachsene Vertrauen der Bevölkerung in die Kanzlerin; ihre exzellenten Medienkontakte, die eine Tabuisierung kritischer Fragen erleichterte; die von ihr lange gehegte und gepflegte (und schließlich bitter enttäuschte) Hoffnung auf europäische Solidarität in der Flüchtlingsfrage. Diese Liste ließe sich weiter fortsetzen (vgl. hierzu Alexander 2017).

[15] Insgesamt macht der bisherige Verlauf der deutschen Flüchtlingsdebatte deutlich, dass der von Ingo Pies geforderte rationale Diskurs im demokratischen Prozess alles andere als ein Selbstläufer ist. Die »Aufsprengung " ([47]) verfestigter Positionen benötigt aber Zeit. Deshalb kann man die Forderungen von Ingo Pies noch durch ein weiteres an die Politik und die Zivilgesellschaft gerichtetes Gebot ergänzen: »Achtet auf die Bewahrung der zeitlichen Spielräume, ohne die eine rationale Kommunikation in einer Demokratie nicht möglich ist! « Wer aus moralisch noch so überzeugend erscheinenden Gründen dieses Gebot missachtet und sich überrennen lässt, trägt zur Aushöhlung demokratischer Prinzipien bei und gefährdet den sozialen Frieden. Auch das ist ein Hiatus zwischen Handlungsmotiven und Handlungsergebnissen.

\section{Literaturverzeichnis}

Arce M., D. G./Sandler, T. (2005): The Dilemma of the Prisoner's Dilemmas, in: Kyklos, Vol. 58/No. 1: 3-24.

Alexander, R. (2017): Die Getriebenen - Merkel und die Flüchtlingspolitik: Report aus dem Innern der Macht, München: Siedler.

Bloom, P. (2016): Against Empathy - The Case for Rational Compassion, New York: HarperCollins.

Betts, A./Collier, P. (2017): »Gestrandet«. Warum unsere Flüchtlingspolitik allen schadet und was jetzt zu tun ist, München: Siedler.

Brekke, K. A./Kverndokk, S./Nyborg, K. (2003): An Economic Model of Moral Motivation, in: Journal of Public Economics, Vol. 87/No. 9-10: 1967-1983.

Buchbolz, W./Eichenseer, M. (2019): Prisoner's Dilemma, in: Marciano, A./Ramello, G. B. (Eds.): Encyclopedia of Law and Economics, Berlin: Springer, im Erscheinen.

Buchbolz, W./Peters, W./Ufert, A. (2014): Spielräume für uni- und multilateralen Klimaschutz, in: Zeitschrift für Umweltpolitik \& Umweltrecht, Jg. 37/H. 3: 326-344.

Dohmen, T./Falk, A./Huffman, D./Sunde, U. (2009): Homo Reciprocans: Survey Evidence on Behavioural Outcomes, in: Economic Journal, Vol. 119/No. 536: 592-612.

Downs, A. (1972): Up and Down with Ecology - The "Issue-Attention Cycle«, in: Public Interest, Vol. 28, 38-50. 
Fischbacher, U./Gächter, S./Fehr, E. (2001): Are People Conditionally Cooperative? Evidence from a Public Goods Experiment, in: Economics Letters, Vol. 71: 397-404.

Grundmann, T./Stephan, A. (Hrsg.) (2016): »Welche und wie viele Flüchtlinge sollen wir aufnehmen? « - Philosophische Essays, Stuttgart: Reclam.

Hobbes, T. (1996 [1651]): Leviathan, hrsg. von G.C.A. Gaskin, Oxford: Oxford University Press.

Janisch, W. (2017): Ein Urteil ohne Kreativität und Herz, in: Süddeutsche Zeitung, 7. März 2017.

Pittel, K./Rübbelke, D. (2013): International Climate Finance and its Influence on Fairness and Policy, in: The World Economy, Vol. 36/No. 4: 419-436.

Rabin, M. (1993): Incorporating Fairness into Game Theory and Economics, in: American Economic Review, Vol. 83/No. 5: 1281-1302.

Roemer, J. (2015): Kantian Optimization: A Microfoundation for Cooperation, in: Journal of Public Economics, Vol. 127: 45-57.

Ufert, A. E. (2016): Reciprocity in Climate Change Policy - How to Solve the Social Dilemma of Climate Protection, Frankfurt (Oder): Europa-Universität Viadrina, Dissertation.

Van Long, N. (2017): Mixed Strategy Kant-Nash Equilibrium and Private Contributions to a Public Good, in: Buchholz, W./Rübbelke, D. (Eds.): The Theory of Externalities and Public Goods - Essays in the Memory of Richard C. Cornes, Cham CH: Springer, 107126.

\title{
Biodiversität und die Voraussetzungen eines guten Lebens
}

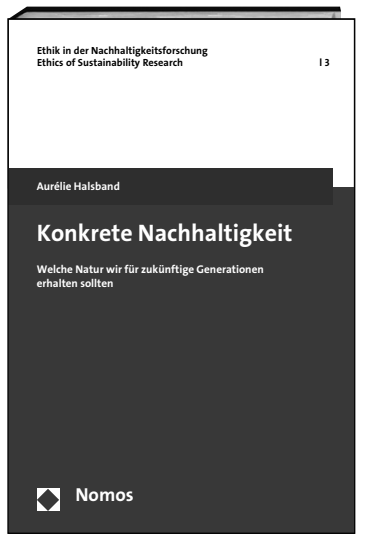

\author{
Konkrete Nachhaltigkeit \\ Welche Natur wir für zukünftige \\ Generationen erhalten sollten \\ Von Dr. Aurélie Halsband \\ 2016, 326 S., brosch., 59,-€ \\ ISBN 978-3-8487-3464-1 \\ eISBN 978-3-8452-7834-6
}

(Ethik in der Nachhaltigkeitsforschung /

Ethics of Sustainability Research, Bd. 3)

nomos-shop.de/28165

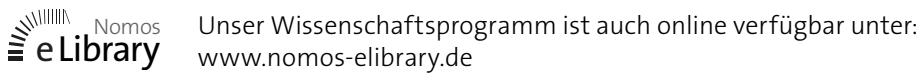

Portofreie Buch-Bestellungen unter www.nomos-shop.de

Alle Preise inkl. Mehrwertsteuer

Nomos 